\title{
Trajectory of post-traumatic stress following traumatic injury: 6-year follow-up
}

Richard A. Bryant, Angela Nickerson, Mark Creamer, Meaghan O’Donnell, David Forbes, Isaac Galatzer-Levy, Alexander C. McFarlane and Derrick Silove

\section{Background}

Traumatic injuries affect millions of patients each year, and resulting post-traumatic stress disorder (PTSD) significantly contributes to subsequent impairment.

\section{Aims \\ To map the distinctive long-term trajectories of PTSD responses over 6 years by using latent growth mixture modelling.}

\section{Method}

Randomly selected injury patients $(n=1084)$ admitted to four hospitals around Australia were assessed in hospital, and at 3, 12, 24 and 72 months. Lifetime psychiatric history and current PTSD severity and functioning were assessed.

\section{Results}

Five trajectories of PTSD response were noted across the 6 years: (a) chronic (4\%), (b) recovery (6\%), (c) worsening/ recovery (8\%), (d) worsening (10\%) and (e) resilient (73\%). A poorer trajectory was predicted by female gender, recent life stressors, presence of mild traumatic brain injury and admission to intensive care unit.

\section{Conclusions}

These findings demonstrate the long-term PTSD effects that can occur following traumatic injury. The different trajectories highlight that monitoring a subset of patients over time is probably a more accurate means of identifying PTSD rather than relying on factors that can be assessed during hospital admission.

\section{Declaration of interest}

None.

\section{Copyright and usage}

(c) The Royal college of Psychiatrists 2015.
Traumatic injuries affect millions of people each year, and cause marked impairment. For example, road traffic traumatic injuries are projected to be the third largest burden of disease globally by $2020 .^{1}$ These injuries often result in psychological injury and particularly post-traumatic stress disorder (PTSD). ${ }^{2}$ One study of 2931 injury patients found that $23 \%$ developed PTSD at 12 months after the injury. ${ }^{3}$ One of the major limitations of previous research on psychological effects of injury is the focus on the diagnostic category of PTSD. This is limited because it presumes that the PTSD diagnosis adequately captures those patients with impairment. However, subsyndromal levels of PTSD can contribute to marked impairment ${ }^{4}$ indicating that it is more important to study the severity of symptoms than the diagnostic category. In recent years latent growth mixture modelling (LGMM) has been applied to studying PTSD responses following traumatic events. ${ }^{5}$ This approach classifies homogenous groups in a population to identify class of individual variation over time. The growth curves can be modelled separately, which permits identification of different trajectories of response. Most studies of PTSD trajectory have identified four major trajectories following traumatic experience: (a) resilient class with consistently few PTSD symptoms (also referred to as 'resistant' by some researchers ${ }^{6}$ ), (b) recovery with initial distress then gradual remission over time, (c) delayed reaction with worsening symptoms over time, and (d) chronic distress with consistently high PTSD levels. These patterns have been found across different trauma populations. ${ }^{7,8}$ The limitation of these studies to date is the short-term nature of the assessments; the majority have limited their study to 1 year after the trauma and the longest trajectory analysis of traumatic injury survivors is 6 months. ${ }^{9}$ Accordingly, this study reports the largest, and longest, multisite analysis of PTSD reactions following traumatic injury that indexed PTSD reactions in hospital following traumatic injury and again at $3,12,24$ and 72 months after injury.

\section{Method}

\section{Participants}

These data were collected as part of a 6-year follow-up of injury patients recruited from four hospitals in three states of Australia (in the period April 2004 to February 2006). All patients were admitted into the trauma service for at least $24 \mathrm{~h}$ and met the DSM-IV Criterion A1. ${ }^{10}$ Approval for this study was gained from the Human Research Ethics Committee (HREC) at each hospital. Patients were recruited to the study if they had experienced a traumatic injury (following motor vehicle accident, assault, traumatic fall or work injury) that required a hospital admission of greater than $24 \mathrm{~h}$, were aged between 16 and 70 years, and had sufficient English comprehension to complete the assessment. Patients were excluded if they experienced a moderate to severe traumatic brain injury, or were currently suicidal or psychotic.

Weekday trauma service admissions over a 22-month period were randomly selected. Only weekday admissions were included because of limitations in recruitment resources. Random selection occurred through an automated procedure, stratified by length of stay; this was done because the numbers of patients admitted to each trauma service was far greater than the study's recruitment resources allowed. Of the 1590 patients identified for the study, $1084(68 \%)$ consented to participate. At 72 months 613 (56.5\% of participants) completed the assessment. Of the 1084 initially assessed, $437(40 \%)$ sustained a mild traumatic brain injury (mTBI), which was defined as acute brain injury resulting from external insult to the head and confusion or disorientation, loss of consciousness for $30 \mathrm{~min}$ or less, post-traumatic amnesia for less than $24 \mathrm{~h}$ and a Glasgow Coma Scale (GCS) of 13 to 15 after 30 min. ${ }^{11}$ Participant characteristics, according to mTBI status, are presented in Table 1. 


\begin{tabular}{|c|c|c|c|}
\hline & $\begin{array}{l}\text { Total, \% (n) } \\
(n=1084)\end{array}$ & $\begin{array}{l}\text { Mild traumatic brain injury, \% (n) } \\
\qquad(n=437)\end{array}$ & $\begin{array}{l}\text { Non-mild traumatic brain injury, \% (n) } \\
\qquad(n=647)\end{array}$ \\
\hline \multicolumn{4}{|l|}{ Gender } \\
\hline Male & $72.7(788)$ & $71.7(310)$ & 73.9 (478) \\
\hline Female & $27.3(296)$ & $28.3(127)$ & $26.1(169)$ \\
\hline \multicolumn{4}{|l|}{ Age } \\
\hline $18-24$ & $21.2(230)$ & $26.8(117)$ & $17.5(113)$ \\
\hline $25-34$ & $23.3(253)$ & 24.7 (108) & $22.4(145)$ \\
\hline $35-44$ & $22.6(245)$ & $20.6(90)$ & $24.0(155)$ \\
\hline $45-54$ & $19.4(210)$ & $16.7(73)$ & $21.2(137)$ \\
\hline $55-64$ & $11.2(121)$ & $10.3(45)$ & $11.7(76)$ \\
\hline $65+$ & $2.3(25)$ & 0.9 (4) & $3.2(21)$ \\
\hline \multicolumn{4}{|l|}{ Type of injury } \\
\hline Transport & 64.5 (699) & 75.9 (332) & $56.7(367)$ \\
\hline Assault & $6.1(66)$ & $8.7(38)$ & $4.3(28)$ \\
\hline Traumatic fall & $14.4(156)$ & $7.8(34)$ & $18.9(122)$ \\
\hline Work injury & $7.2(78)$ & $2.5(11)$ & $10.4(67)$ \\
\hline Other injury & $7.8(85)$ & $5.0(22)$ & 9.7 (63) \\
\hline \multicolumn{4}{|l|}{ Injury Severity Score } \\
\hline Minimum & $7.5(81)$ & $4.8(21)$ & $9.3(60)$ \\
\hline Moderate & $28.8(312)$ & $25.4(111)$ & 31.1 (201) \\
\hline Severe & $44.2(479)$ & $38.0(166)$ & $48.4(313)$ \\
\hline Serious & $13.4(145)$ & $21.3(93)$ & $8.2(53)$ \\
\hline Critical & $6.1(66)$ & $10.5(46)$ & $3.0(20)$ \\
\hline \multicolumn{4}{|l|}{ Ethnic status } \\
\hline White & $87.6(950)$ & $86.3(377)$ & $86.2(558)$ \\
\hline Other & $12.4(134)$ & $13.7(60)$ & $13.8(89)$ \\
\hline \multicolumn{4}{|l|}{ Marital status } \\
\hline Married/de facto & $48.2(522)$ & $48.5(212)$ & $47.9(310)$ \\
\hline Single & $51.9(562)$ & $51.5(225)$ & $52.1(337)$ \\
\hline \multicolumn{4}{|l|}{ Employment status } \\
\hline Employed & $78.8(854)$ & $79.2(346)$ & $78.5(508)$ \\
\hline Unemployed & $6.0(65)$ & $5.7(25)$ & $6.2(40)$ \\
\hline Not in labour ${ }^{\mathrm{a}}$ & $15.2(165)$ & $15.1(66)$ & $15.3(99)$ \\
\hline \multicolumn{4}{|l|}{ Education } \\
\hline Bachelor degree or higher & $17.2(186)$ & $16.7(73)$ & $17.5(113)$ \\
\hline Diploma & $5.4(58)$ & $5.0(22)$ & $5.6(36)$ \\
\hline Trade & $36.5(396)$ & $36.8(161)$ & $36.3(235)$ \\
\hline High school & 41.0 (444) & 41.5 (181) & $40.6(263)$ \\
\hline
\end{tabular}

Of those who completed the 6-year assessment, the majority were male $(72 \% ; n=441)$ with an average age of 40 years (s.d.=13.48). Half the sample was married or in a de facto relationship ( $53 \% ; n=325)$. On average patients spent 13.32 days (s.d. $=14.61)$ in hospital. In total, $43 \%$ of participants sustained a mTBI $(n=236)$ and the mean Injury Severity Score (ISS) ${ }^{12}$ was 11.12 (s.d. =7.80), which is in the moderate range of severity. The principal mechanism of injury was transport accident $(66 \%$, $n=407)$, followed by traumatic falls $(16 \%, n=97)$, assault $(6 \%$, $n=36$ ), work-related accidents not specified in the above categories (5\%, $n=30)$ and other (such as electrocution, mudslides and rockfalls, and near-drownings; 7\%, $n=43$ ).

Individuals who refused to participate in the study did not differ from participants in gender, the presence of a mTBI, education, mechanism of injury, length of stay or ISS. Those who did not complete the 72-month assessment did not differ from those who were recruited in terms of gender, the presence of a mTBI, education, mechanism of injury, length of stay or ISS. Those who did not complete the 6-year assessment differed from completers in that they were more likely to be younger (mean 36.33 (s.d.=13.56) v. 39.53 (s.d.=13.48), $(t(1126)=3.97, P<0.001)$ and have higher baseline ClinicianAdministered PTSD Scale for DSM-IV (CAPS) ${ }^{13}$ scores (mean $20.21 \quad$ (s.d. $=17.89) \quad$ v. 16.13, $\quad($ s.d. $=15.06), \quad(t(1113.9)=4.17$, $P<0.001)$.

\section{Procedures}

The study procedures were fully explained to the patients who met inclusion criteria and written informed consent was obtained. Whereas the baseline assessment was conducted in person in hospital, each subsequent assessment was conducted via telephone to determine psychiatric diagnosis and self-report questionnaires with prepaid return envelopes were sent to participants to assess disability and quality of life.

\section{Measures}

PTSD

Symptom severity of PTSD and diagnosis were assessed using CAPS. ${ }^{13}$ This structured clinical interview is one of the most widely used tools for diagnosing PTSD and has demonstrated excellent reliability and validity. ${ }^{14}$ The CAPS interviews were conducted via telephone, a method that has been shown to be as valid and reliable as face-to-face interviews. ${ }^{15}$ All interviews were digitally recorded to ensure ongoing adherence to the protocol. To test interrater reliability, 5\% of all CAPS interviews were assessed by an independent assessor who was masked to the original scoring. Overall, the diagnostic consistency on the CAPS was $100 \%$. 


\section{Lifetime psychiatric disorder}

The Mini International Neuropsychiatric Interview Version 5.5 (MINI) ${ }^{16}$ was used at the baseline assessment to assess a diagnosis of major depressive episode, panic disorder, agoraphobia, social phobia, obsessive-compulsive disorder, generalised anxiety disorder, alcohol misuse and dependence, and marijuana misuse and dependence. The MINI is a short, structured diagnostic interview based on the DSM-IV and the ICD- $10^{17}$ classification of mental illness, especially designed to be used in clinical trials and epidemiological studies. The MINI consists of a set of screening questions and modules; modules are administered if a patient responds positively to the screening question. The MINI has good reliability for all diagnosis when compared with the CIDI. $^{16}$

\section{Functional outcomes}

We used the psychological domain scale from the World Health Organization Quality of Life - Bref (WHOQOL-Bref) ${ }^{18}$ to measure quality of life. The WHOQOL-Bref psychological domain is an eight-item scale that assesses quality of life in terms of perception and satisfaction across a number of life areas. The WHOQOL-Bref demonstrates good discriminant validity, content validity, internal consistency and test-retest reliability. ${ }^{18}$ In the current study, a scoring algorithm was used to standardise scores to a 0-100 scale with higher scores indicating higher quality of life. Australian population norms were used to identify thresholds and a score of 55.5 was used as the cut-off for poor psychological quality of life. ${ }^{19}$

\section{Stressful life events}

Subsequent aversive events were assessed by an adaptation of the Recent Life Events Questionnaire. ${ }^{20}$ The RLEQ indexes the occurrence of 20 common stressful life events that encompass both traumatic (for example, assaults) and aversive (for example, losing one's job) events.

\section{Data analysis}

We employed latent class growth analysis (LCGA) to examine PTSD trajectories, using Mplus version 6.2 for Windows. ${ }^{21}$ LCGA models heterogeneity in longitudinal data by identifying symptom trajectories across time. Mplus employs a robust full information maximum likelihood estimation procedure to account for missing data. We first identified the best-fitting unconditional trajectory model by comparing the model fit of progressive numbers of classes. After finding the best fitting unconditional model, we integrated covariates into this model (conditional model). The following variables were examined on the basis of evidence that they influence risk for PTSD: gender, age at admission, heart rate upon admission, respiration rate upon admission, ISS, mTBI, admission into the ICU and psychiatric history. ${ }^{22}$ Only variables that significantly predicted class membership were retained (see online supplement).

We then tested a series of nested models using the five-class solution to identify covariates that improved model fit. We tested the same covariates listed above, as well as recent stressful life events at 3 months, 12 months and 24 months. Only covariates that were significant predictors of class membership and improved model fit were retained in the final solution. We used multiple imputation in MPlus to impute missing values of covariates. Logistic regression analyses were conducted to identify predictors of class membership for the five-class conditional model (see Table 3).

\section{Results}

\section{Unconditional model}

Figure 1 displays the trajectories identified in the five-class solution. Individuals in the chronic class (4\%) displayed a pattern of initially high levels of PTSD symptoms that increase over the subsequent 24 months, and remain elevated by the 6 year measurement. Those in the recovery class $(6 \%)$ displayed a pattern of high levels of PTSD symptoms initially that reduce consistently over the subsequent 6 years. Participants in the worsening/ recovery class $(8 \%)$ displayed a pattern of relatively low initial symptoms of PTSD that increase over the subsequent 24 months before returning to their initial level by the 6-year measurement. Individuals in the worsening class (10\%) displayed a trajectory in which participants report relatively low levels of PTSD symptoms at baseline but these increase gradually over the subsequent 6 years. Finally, those in the resilient class $(73 \%)$ displayed consistently low levels of PTSD symptoms over the 6 years of the study (see online supplement).

\section{Functional impairment}

Table 2 presents the odds ratios of impaired functioning (as indexed by the WHOQOL) for each trajectory class at 3, 12, 24 months and 6 years. Notably, each of the chronic, worsening, recovery and worsening/recovery classes displayed more impaired functioning across the different functioning domains than the resilient class over the initial 2 years. Interesting patterns emerged at the 6-year assessment, however, when the recovery and worsening/recovery class showed less impairment. The recovery class was no more impaired than the resilient class by 6 years. Although the worsening/recovery class was still more impaired than the resilient class at 6 years, the extent of this difference had diminished markedly relative to the previous assessments; the odds ratios of impaired functioning in the worsening/recovery class were at least one-third of those at the 24- and 12-month assessments.

\section{Conditional model}

Following the unconditional model, we examined a series of nested five-class models to investigate the impact of the inclusion of covariates on model fit. Significant covariates included gender, mTBI and life events at 3 months, 12 months and 24 months following trauma exposure. Model fit was improved by the inclusion of these covariates. The structure of the five-class solution remained consistent between the unconditional and conditional models. Class membership proportions, and intercept, linear slope and quadratic slope parameters for each class are presented in online Table DS2 for the unconditional and conditional models. Class membership remained largely consistent across the unconditional and conditional models (Chronic unconditional $5.2 \% \quad v$. conditional $5.2 \%$; worsening/recovery unconditional $8.1 \% v$. conditional $8.1 \%$, worsening unconditional $9.6 \%$ v. conditional $10.2 \%$, recovery unconditional $5.7 \% \quad v$. conditional $8.2 \%$, resilient unconditional $72.3 \% \quad v$. conditional $68.3 \%)$. Similarly, the magnitude and significance of intercept, linear slope and quadratic slope parameters remained consistent across the unconditional and conditional models, with the exception of the quadratic slope component of the resilient class, which was statistically significant in the unconditional model, but not in the conditional model.

\section{Covariate predictors of trajectory class}

Female gender was associated with membership in the chronic, recovered, and worsening classes (relative to the resilient class; Table 3). Membership in the chronic, worsening/recovery and 


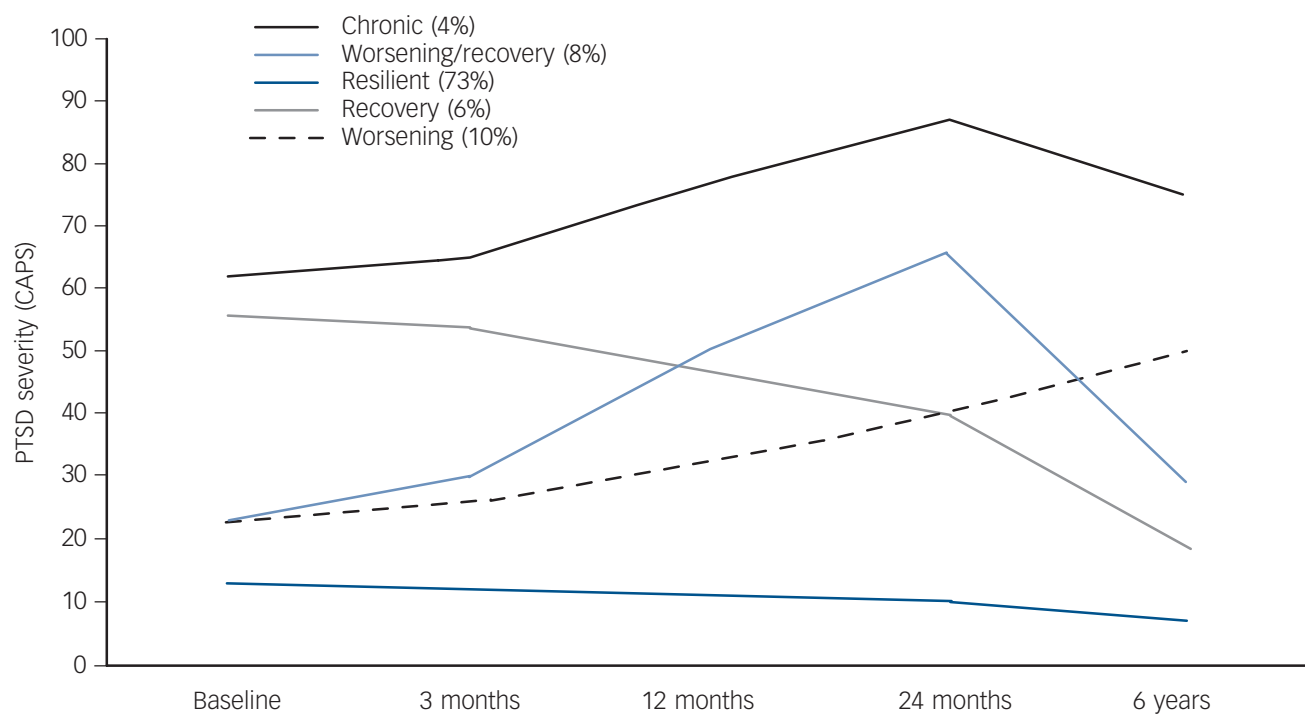

Fig. 1 Trajectories of post-traumatic stress disorder (PTSD) response after traumatic injury.

Patients followed five distinct trajectories: a chronic class (4\%), a recovery class (6\%), a worsening/recovery class (8\%), a worsening class (10\%) and a resilient class $(73 \%)$. CAPS, Clinician-Administered PTSD Scale for DSM-IV.

\begin{tabular}{|c|c|c|c|c|}
\hline & \multicolumn{4}{|c|}{ Class, odds ratio $(95 \% \mathrm{Cl})$} \\
\hline & Chronic & Worsening & Recovery & Worsening/recovery \\
\hline \multicolumn{5}{|l|}{3 months } \\
\hline Physical & $4.12 *(2.51-6.75)$ & $1.38 *(1.16-1.63)$ & $5.87 *(3.09-11.14)$ & $1.50 *(1.31-1.71)$ \\
\hline Psychological & $39.50 *(5.60-187.32)$ & $1.39 *(1.15-1.68)$ & $29.69 *(4.06-217.23)$ & $1.77(1.40-2.23)$ \\
\hline Social & $3.03 *(1.89-4.85)$ & $1.38 *(1.16-1.64)$ & $6.79 *(3.16-14.60)$ & $1.51 *(1.31-1.75)$ \\
\hline Environmental & $5.32 *(2.58-10.99)$ & $1.46 *(1.24-1.72)$ & $6.42 *(3.17-12.99)$ & $1.47 *(1.29-1.69)$ \\
\hline \multicolumn{5}{|l|}{12 months } \\
\hline Physical & $5.23 *(3.31-8.26)$ & $1.79 *(1.48-2.17)$ & $7.98 *(3.87-16.42)$ & $1.87 *(1.61-2.17)$ \\
\hline Psychological & $6.17 *(3.34-11.39)$ & $1.90 *(1.59-2.27)$ & $27.61 *(10.45-72.88)$ & $1.97 *(1.67-2.32)$ \\
\hline Social & $3.77 *(2.19-6.46)$ & $1.59 *(1.34-1.90)$ & $6.44 *(3.02-13.72)$ & $1.65^{*}(1.41-1.93)$ \\
\hline Environmental & $4.07 *(2.37-6.99)$ & $1.60 *(1.34-1.90)$ & $7.16^{*}(3.36-15.26)$ & $1.65^{*}(1.41-1.94)$ \\
\hline \multicolumn{5}{|l|}{24 months } \\
\hline Physical & $7.09 *(3.97-12.68)$ & $2.06 *(1.69-2.50)$ & $5.21 *(2.17-12.50)$ & $2.05 *(1.75-2.41)$ \\
\hline Psychological & $5.08 *(2.71-9.55)$ & $1.98 *(1.65-2.36)$ & $8.99 *(4.13-19.53)$ & $2.30 *(1.89-2.81)$ \\
\hline Social & $3.26 *(1.74-6.09)$ & $1.49 *(1.25-1.77)$ & $4.35 *(1.96-9.50)$ & $1.72 *(1.43-2.05)$ \\
\hline Environmental & $5.44 *(2.59-11.42)$ & $1.69 *(1.42-2.01)$ & $4.92 *(2.32-10.42)$ & $1.74 *(1.48-2.04)$ \\
\hline \multicolumn{5}{|l|}{6 years } \\
\hline Physical & $5.89 *(2.74-12.68)$ & $1.97 *(1.62-2.40)$ & $2.01(0.70-5.73)$ & $1.39 *(0.98-1.52)$ \\
\hline Psychological & $5.02 *(2.34-10.77)$ & $1.99 *(1.63-2.42)$ & $2.40(0.93-6.19)$ & $1.31 * *(1.10-1.56)$ \\
\hline Social & $3.49 *(1.62-7.48)$ & $1.56^{*}(1.28-1.89)$ & $3.21^{* *}(1.31-7.86)$ & $1.34^{*}(1.14-1.61)$ \\
\hline Environmental & $3.08 *(1.69-5.63)$ & $1.99 *(1.64-2.43)$ & $4.83(1.97-11.81)$ & $1.36 *(1.14-1.62)$ \\
\hline
\end{tabular}

worsening classes was predicted (relative to the resilient class) by the presence of mTBI at the time of injury. Stressful recent life events at 3 months predicted membership in the chronic, recovered and worsening recovery classes (relative to the resilient class). Stressful recent life events at 12 months predicted membership in all of the symptomatic classes, compared with the resilient class. Stressful recent life events at 24 months predicted membership in the chronic and worsening/recovery classes, relative to the resilient class. Female gender predicted membership in the chronic and resilient classes, compared with the worsening/recovery class.

\section{Mental health service utilisation}

A one-way analysis of variance (ANOVA) of visits to mental health professionals between trajectory classes indicated a significant effect $(F(4,611)=20.53, P=0.000)$. Participants in the chronic class (mean 7.16, s.d. $=9.76$ ) and the worsening class (mean 4.02 , s.d. $=6.53$ ) reported more mental health visits than those in the worsening/recovery class (mean 2.57, s.d.=5.77) and recovery class (mean 1.05 , s.d. $=2.80$ ), who in turn had more visits than those in the resilient class (mean 0.70, s.d. $=2.29$ ).

\section{Discussion}

\section{Main findings}

This study found five different trajectories of PTSD response over the 6 years after injury. Consistent with previous studies, we observed a chronic trajectory that maintained elevated PTSD symptoms over 6 years, a resilient group that had very few 
Table 3 Baseline predictors of trajectory membership

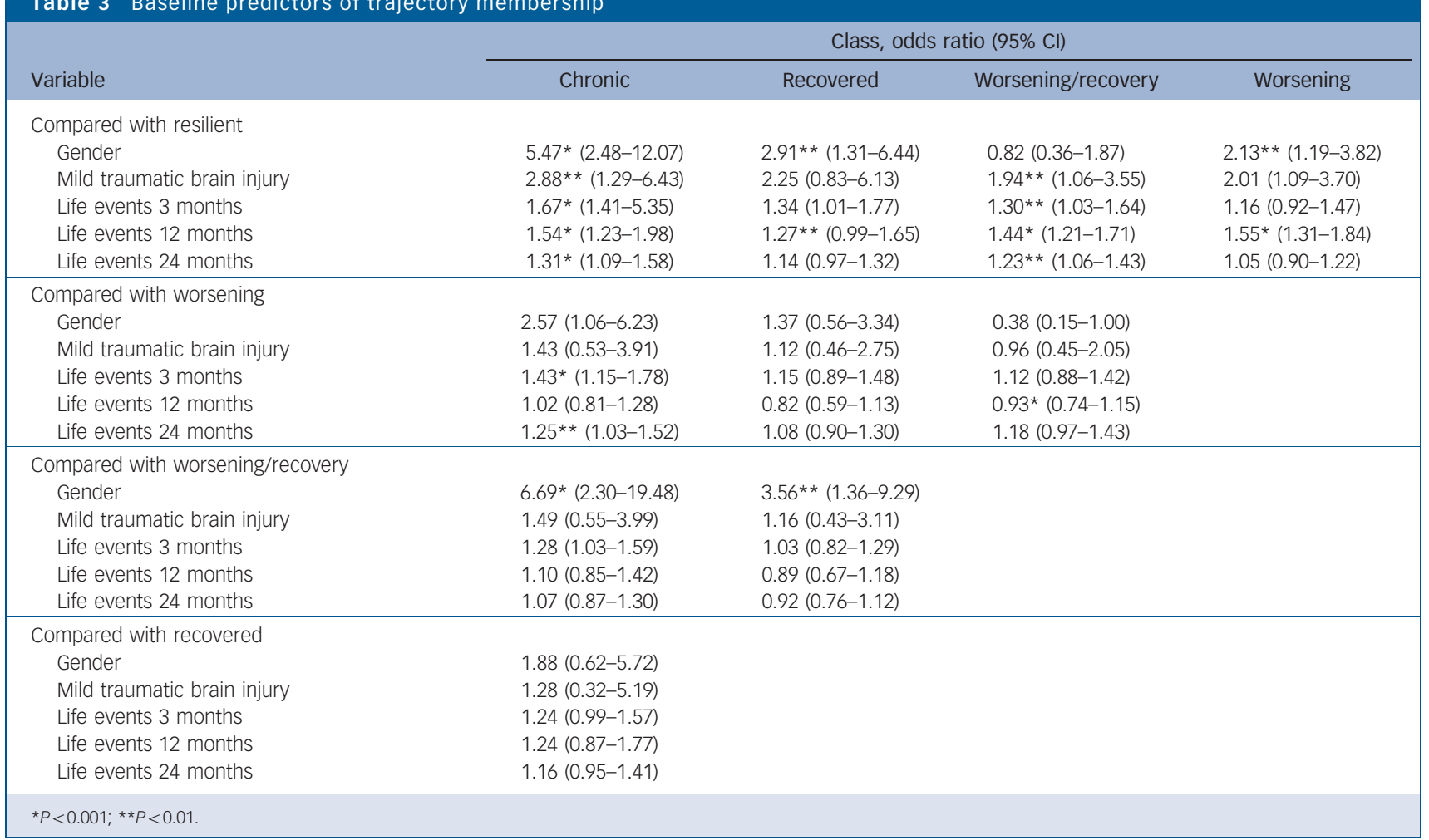

symptoms at any point, a recovery trajectory that showed initial symptoms that eased over time, and a delayed or worsening trajectory. In addition, and unlike previous studies, we observed a group of patients who worsened over time but then recovered (worsening/recovery).

The most concerning finding of this study is that a small, but significant, proportion of trauma survivors display persistently chronic stress reactions. The chronic class showed no signs of remitting, even after 6 years, and suggests that they are embarking on a very long-term course of PTSD. Worryingly, this group had more overall psychological impairment, as measured by the WHOQOL, than any other group at each assessment. This pattern suggests that this subgroup of injury survivors is likely to experience long-term impairment. The other curious finding, in those with a recovery trajectory, was that they had comparably impaired functioning relative to those in the chronic class. This points to the possibility that having experienced more severe PTSD this left residual impairments despite the remission of symptoms; this interpretation is consistent with findings that comparable levels of impairment are found in people with full and subsyndromal PTSD., ${ }^{4,23}$

\section{Comparison with findings from other studies}

The most novel aspect of this study was it followed up trauma survivors at least 3 years longer than previous studies. By adopting this approach, we were able to demonstrate that nearly one-tenth of the cohort who appeared to be moving on a trajectory towards worsening PTSD 2 years after the injury, subsequently recovered by 6 years. This suggests that it will take some trauma survivors considerably longer to adapt to the injury than others, even though it appears in the initial years that they are on a worsening trajectory. This observation contrasts with previous studies that have ceased assessing patients at shorter time frames since the trauma. Whereas these studies conclude that a subgroup of trauma survivors will be on a course of worsening symptoms, ${ }^{9,24}$ our longer-term follow-up suggests that these short-term followups portray an incomplete profile of adaptation because many of these patients who are initially worsening over time do eventually recover.

\section{Identifying those most at risk of long-term mental health problems}

One of the challenges raised by the current findings is how early detection can be achieved in the acute phase after traumatic injury to identify the subsequent trajectory that patients will follow. Figure 1 highlights that in hospital there were essentially two groups of patients: those with elevated PTSD levels (including those belonging to the chronic and recovery classes) and those with low PTSD levels (including those belonging to the resilient, worsening and worsening/recovery classes). The current findings underscore how mental health outcomes cannot necessarily be predicted on the basis of severity of injury, as injury severity score was not a consistent predictor of subsequent outcome. Prior attempts to identify longer-term adaptation after trauma have been modest. ${ }^{25}$ However, admission to ICU did differentiate the chronic from resilient class, suggesting that ICU admission may be one potential marker of those who are more at risk of longterm PTSD symptoms. The current findings highlight that a major reason for this difficulty in accurate prediction of outcome of psychological state is that there is not a linear relationship between acute and longer-term responses.

In terms of predictors of trajectory classes, mTBI may contribute to worse outcomes following trauma for several possible reasons. The prevailing models of PTSD postulate that the disorder is maintained because of impaired regulation of the amygdala by the ventral medial prefrontal cortex. ${ }^{26}$ The prefrontal cortex is commonly impaired in mTBI and so this damage may compromise the capacity of patients to manage the emotional sequelae and cognitive demands of the traumatic experience. ${ }^{27}$ 
This finding suggests that patients with mTBI should be considered at higher risk for one of the trajectories that will lead to longer-term psychological problems following traumatic injury.

The finding that stressful life events since the trauma characterised poor adjustment at each assessment accords with evidence that post-trauma stressors are associated with PTSD. ${ }^{28}$ Fear-reinstatement models explain this finding in terms of subsequent stressors reactivating previously conditioned trauma responses. ${ }^{29}$ Sensitisation models posit that trauma can sensitise neural circuitry, which then leads to excessive stress responses to aversive events. ${ }^{30}$ Further, resource models account for this pattern in terms of additional stressors diminishing limited resources following trauma, and this compounds the PTSD reaction. ${ }^{31}$ In any case, these findings highlight that the trajectory one follows after trauma will be influenced by ongoing stressors.

The pattern of female gender being associated with more distressed trajectory classes is consistent with evidence that females are markedly more at risk of PTSD than males. ${ }^{22}$ One curious finding was that female gender predicted membership of the resilient class relative to the worsening/recovery class. The worsening/recovery class, which has not been previously identified in trajectory analyses, reflects a course in which there is a timelimited period of worsening distress followed by good mental health. This trajectory is poorly understood and it is difficult to understand why females are more likely to be included in the resilient class than in the class that is worsening and then recovering. It is possible that worsening symptoms followed by recovery reflects a distinctive pathway that is less susceptible to the expected influences of gender. This course requires further study to understand the predictors of this long-term recovery. In this context, we also note that it is possible that some traumatic injury patients may have a worsening trajectory during the protracted period of physical injury, pain and rehabilitation, followed by remission of symptoms as they progress beyond this period.

Three of the trajectories were characterised by a worsening of symptoms over the initial year after traumatic injury: the chronic, worsening, and worsening/recovery classes. This observation is consistent with sensitisation models of PTSD, which propose a vulnerability to excessive responses to less stressful events following traumatic exposure because neural circuitry is sensitised by the initial traumatic experience. ${ }^{32}$ This model accords with evidence that prior trauma is linked to more reactive responses to subsequent stressors. ${ }^{33}$ There is also evidence that trauma survivors who subsequently develop PTSD do not display elevated startle responses immediately after the traumatic event but do so after several months have elapsed. ${ }^{34}$ It is possible that the psychological effects of the traumatic injury may not be fully experienced by survivors in the initial period, however, the subsequent stressors that are often faced by injury survivors may compound initial reactions via sensitisation processes, thereby leading to more severe PTSD responses in the following year. This conclusion remains speculative because to address this issue directly, studies need to model ongoing stressors in terms of how people can transfer between classes over time (via latent transition analysis).

\section{Limitations}

We recognise several limitations. First, nearly half the sample was lost by the 6-year assessment, and these participants were younger and had more severe initial PTSD at baseline than those who were retained; this differential drop-out may have affected results. Second, the cohort comprised injury survivors who had been admitted to hospital, and so the findings need to be confirmed in samples exposed to different types of trauma. Third, we assessed PTSD at each follow-up by structured clinical interview via the telephone rather than face-to-face interview; we note, however, that there is very strong concordance between these two formats. ${ }^{15}$ Fourth, this was a largely male sample and these results may not generalise to females. Fifth, future research could usefully index utilisation of general health services to map how people in different trajectories access services, including profiling of health economics associated with each class.

\section{Implications}

The major outcome of this study is that it highlights the long-term psychological dysfunction and associated impairment, experienced by a proportion of traumatically injured patients. One implication of the study is that by identifying the majority of those who are resilient, primary care facilities may be able to triage the remainder of patients to a category that may benefit from either early intervention or subsequent monitoring to index their mental health. Further work is required, however, to develop the means to discriminate between injured patients in the hospital setting who are most likely to enjoy a resilient trajectory and those who at some time may benefit from mental health assistance.

Richard A. Bryant, PhD, School of Psychology, University of New South Wales and Brain Dynamics Centre, University of Sydney and Westmead Millennium Institute Sydney, Australia; Angela Nickerson, PhD, School of Psychology, University of New South Wales, New South Wales, Australia; Mark Creamer, PhD, Meaghan o'Donnell, PhD, David Forbes, PhD, Australian Centre for Posttraumatic Mental Health, Department of Psychiatry, University of Melbourne, Melbourne; Isaac Galatzer-Levy, PhD, Department of Psychiatry, New York University, New

York, USA; Alexander C. McFarlane, MD, Centre for Military and Veteran Health, York, USA; Alexander C. McFarlane, MD, Centre for Military and Veteran Health,
University of Adelaide, Adelaide, Australia; Derrick Silove, MD, School of Psychiatry, University of New South Wales, New South Wales, Australia

Correspondence: Richard Bryant, School of Psychology, University of New South Wales, New South Wales 2052, Australia. Email: r.bryant@unsw.edu.au

First received 24 Jan 2014, final revision 11 Jul 2014, accepted 27 Jul 2014

\section{References}

1 Murray CJ, Lopez AD. Alternative projections of mortality and disability by cause 1990-2020: Global Burden of Disease Study. Lancet 1997; 349: 1498-504.

2 Bryant RA, Creamer M, O'Donnell M, Silove D, Clark CR, McFarlane AC. The psychiatric sequelae of traumatic injury. Am J Psychiatry 2010; 167 $312-20$

3 Zatzick DF, Rivara FP, Nathens $A B$, Jurkovich GJ, Wang J, Fan $M-Y$, et al. A nationwide US study of post-traumatic stress after hospitalization for physical injury. Psychol Med 2007; 37: 1469-80.

4 Stein MB, Walker JR, Hazen AL, Forde DR. Full and partial posttraumatic stress disorder: findings from a community survey. Am J Psychiatry 1997; 154: 1114-9.

5 Muthén B. Latent variable analysis: growth mixture modeling and related techniques for longitudinal data. In Handbook of Quantitative Methodology for the Social Sciences (ed D Kaplan): 345-68. Sage, 2004.

6 Norris FH, Tracy M, Galea S. Looking for resilience: understanding the longitudinal trajectories of responses to stress. Soc Sci Med 2009; 68: 2190-8.

7 Pietrzak RH, Van Ness PH, Fried TR, Galea S, Norris FH. Trajectories of posttraumatic stress symptomatology in older persons affected by a large-magnitude disaster. J Psychiatr Res 2013; 47: 520-6.

8 Bonanno GA, Mancini AD, Horton JL, Powell TM, Leardmann CA, Boyko EJ et al. Trajectories of trauma symptoms and resilience in deployed U.S. military service members: prospective cohort study. Br J Psychiatry 2012; 200: 317-23.

9 deRoon-Cassini TA, Mancini AD, Rusch MD, Bonanno GA. Psychopathology and resilience following traumatic injury: a latent growth mixture model analysis. Rehabil Psychol 2010; 55: 1-11.

10 American Psychiatric Association. Diagnostic and Statistical Manual of Mental Disorder (4th edn) (DSM-IV). APA, 1994. 
11 Carroll L, Cassidy JD, Holm L, Kraus J, Coronado VG. Methodological issues and research recommendations for mild traumatic brain injury: the WHO Collaborating Centre Task Force on Mild Traumatic Brain Injury. J Rehabil Med 2004; 36: 113-25

12 Baker SP, O'Neil B, Haddon W, Long WB. The injury severity score: a method for describing patients with multiple injuries and evaluating emergency care. J Trauma 1974; 14: 187-96.

13 Blake DD, Weathers FW, Nagy LM, Kaloupek DG, Charney DS, Keane TM. Clinician-Administered PTSD Scale for DSM-IV. National Center For Posttraumatic Stress Disorder, 1998

14 Weathers FW, Keane TM, Davidson J. Clinician-administered PTSD scale: a review of the first ten years of research. Depress Anxiety 2001; 13: 132-56.

15 Aziz M, Kenford S. Comparability of telephone and face-to-face interviews in assessing patients with posttraumatic stress disorder. J Psychiatr Pract 2004; 10: 307-13.

16 Sheehan DV, Lecrubier $\mathrm{Y}$, Harnett-Sheehan $\mathrm{K}$, Amorim $\mathrm{P}$, Janavs J, Weiller $\mathrm{E}$, et al. The Mini International Neuropsychiatric Interview (M.I.N.I.): the development and validation of a structured diagnostic psychiatric interview. J Clin Psychiatry 1998; 59 (suppl 20): 22-33.

17 World Health Organization. The ICD-10 Classification of Mental and Behavioural Disorders: Clinical Descriptions and Diagnostic Guidelines. WHO 1992.

18 Harper A, Power M. Development of the World Health Organization WHOQOLBREF quality of life assessment. Psychol Med 1998; 28: 551-8.

19 Hawthorne G, Herrman $\mathrm{H}$, Murphy B. Interpreting the WHOQOL-Brèf: preliminary population norms and effect sizes. Soc Indicat Res 2006; 77 : 37-59.

20 Brugha T, Bebbington $\mathrm{P}$, Tennant $\mathrm{C}$, Hurry J. The list of threatening experiences: a subset of 12 life event categories with considerable long-term contextual threat. Psychol Med 1985; 15: 189-94.

21 Muthén LK, \& Muthén, B.O. Mplus User's Guide. Muthén, \& Muthén, 1998-2011.

22 Brewin CR, Andrews B, Valentine JD. Meta-analysis of risk factors for posttraumatic stress disorder in trauma-exposed adults. J Consult Clin Psychol 2000; 68: 748-66.
23 Zlotnick C, Franklin CL, Zimmerman M. Does "subthreshold" posttraumatic stress disorder have any clinical relevance? Compr Psychiatry 2002; 43: 413-9.

24 Bonanno GA, Kennedy P, Galatzer-Levy IR, Lude P, Elfstrom ML. Trajectories of resilience, depression, and anxiety following spinal cord injury. Rehabil Psychol 2012; 57: 236-47.

25 Bryant RA. Early predictors of posttraumatic stress disorder. Biol Psychiatry 2003; 53: 789-95.

26 Rauch SL, Shin LM, Phelps EA. Neurocircuitry models of posttraumatic stress disorder and extinction: human neuroimaging research-past, present, and future. Biol Psychiatry 2006; 60: 376-82.

27 Bryant RA. Disentangling mild traumatic brain injury and stress reactions. N Engl J Med 2008; 358: 525-7.

28 Smid GE, van der Velden PG, Lensvelt-Mulders GJ, Knipscheer JW, Gersons BP, Kleber RJ. Stress sensitization following a disaster: a prospective study. Psychol Med 2012; 42: 1675-86.

29 Norrholm SD, Jovanovic T, vervliet B, Myers KM, Davis M, Rothbaum BO, et al. Conditioned fear extinction and reinstatement in a human fear-potentiated startle paradigm. Learn Mem 2006; 13: 681-5.

30 Post RM, Weiss SRB, Smith MA. Sensitization and kindling. Implications for the evolving neural substrates of post-traumatic stress disorder. In Neurobiological and Clinical Consequences of Stress: from Normal Adaptation to PTSD (ed MJ Friedman, DS Charney and AY Deutch): 203-24. Lipincott-Raven, 1995.

31 Hobfoll SE, Tracy M, Galea S. The impact of resource loss and traumatic growth on probable PTSD and depression following terrorist attacks. J Traum Stress 2006; 19: 867-78.

32 McFarlane AC. The long-term costs of traumatic stress: intertwined physical and psychological consequences. World Psychiatry 2010; 9: 3-10.

33 Breslau N, Davis GC, Andreski P. Risk factors for PTSD-related traumatic events: a prospective analysis. Am J Psychiatry 1995; 152: 529-35.

34 Shalev AY, Peri T, Brandes D, Freedman S, Orr SP, Pitman RK. Auditory startle response in trauma survivors with posttraumatic stress disorder: A prospective study. Am J Psychiatry 2000; 157: 255-61.

Grief can be thought of as an intrinsic aspect of life. Whether being a response to literal death or symbolic loss, grief is the process of working through relinquishing emotional attachments to the lost object. The grieving person resuscitates the existence of the lost object in the psyche replacing an actual absence with an imaginary presence. This enchanting restoration enables them to comprehend the value of their loss so it becomes cathartic and healing. Thus grief should be permitted to occur naturally without the dependence on medicalisation unless it becomes pathological. However, there may be no therapy to 'cure' grief. 